CREAT. MATH. INFORM.

Volume 25 (2016), No. 2,

Pages $205-213$

\title{
On the reformulated reciprocal degree distance of graphs
}

\author{
K. PATtABiRAmAn and M. VijayARAgAVAN
}

ABSTRACT. The reciprocal degree distance (RDD), defined for a connected graph $G$ as vertex-degree-weighted
sum of the reciprocal distances, that is, $R D D(G)=\sum_{u, v \in V(G)} \frac{(d(u)+d(v))}{d_{G}(u, v)}$. The new graph invariant named reformulated reciprocal degree distance is defined for a connected graph $G$ as $\bar{R}_{t}(G)=\sum_{u, v \in V(G)} \frac{(d(u)+d(v))}{d_{G}(u, v)+t}, t \geq$ 0 . The reformulated reciprocal degree distance is a weight version of the $t$-Harary index, that is, $\bar{H}_{t}(G)=$ $\sum_{u, v \in V(G)} \frac{1}{d_{G}(u, v)+t}, t \geq 0$. In this paper, the reformulated reciprocal degree distance and reciprocal degree distance of disjunction, symmetric difference, Cartesian product of two graphs are obtained. Finally, we obtain the reformulated reciprocal degree distance and reciprocal degree distance of double a graph.

\section{INTRODUCTION}

A topological index of a graph is a real number related to the graph; it does not depend on labeling or pictorial representation of a graph. In theoretical chemistry, molecular structure descriptors (also called topological indices) are used for modeling physicochemical, pharmacologic, toxicologic, biological and other properties of chemical compounds [12]. There are several types of such indices, especially those based on vertex and edge distances. One of the most intensively studied topological indices is the Wiener index; for other related topological indices see $[2,3,4,5,19]$.

All the graphs considered in this paper are simple and connected. For vertices $u, v \in$ $V(G)$, the distance between $u$ and $v$ in $G$, denoted by $d_{G}(u, v)$, is the length of a shortest $(u, v)$-path in $G$ and $d_{G}(v)$ is the degree of a vertex $v \in V(G)$. Let $G$ be a connected graph. Then the Wiener index of $G$ is defined as $W(G)=\frac{1}{2} \sum_{u, v \in V(G)} d_{G}(u, v)$ with the summation going over all pairs of distinct vertices of $G$. Similarly, the Harary index of $G$ is defined as $H(G)=\frac{1}{2} \sum_{u, v \in V(G)} \frac{1}{d_{G}(u, v)}$. Das et al. [8] proposed the second and third Harary index and they extend it to the generalized version of Harary index, namely, the $t$-Harary index, which is defined as $\bar{H}_{t}(G)=\frac{1}{2} \sum_{u, v \in V(G)} \frac{1}{d_{G}(u, v)+t}, t \geq 0$. Also they obtained the bounds for $t$-Harary index of $G$ in terms of Wiener index of $G$.

Dobrynin and Kochetova [9] and Gutman [11] independently proposed a vertex-degreeweighted version of Wiener index of a connected graph $G$ called degree distance, which is defined as $D D(G)=\frac{1}{2} \sum_{u, v \in V(G)}\left(d_{G}(u)+d_{G}(v)\right) d_{G}(u, v)$. Note that the degree distance is a degree-weight version of the Wiener index.

Received: 28.01.2016. In revised form: 08.05.2016. Accepted: 26.05.2016

2010 Mathematics Subject Classification. 05C12, 05 C76.

Key words and phrases. Reformulated reciprocal degree distance, reciprocal degree distance, graph operation.

Corresponding author: K. Pattabiraman; pramank@gmail.com 
To strengthen the interactions between nodes in a network is described by their topological distances, it is necessary to consider the weighted versions to measure the centrality of the network with respect to the information flow [6]. Hua and Zhang [13] introduced a new graph invariant named reciprocal degree distance, which can be seen as a degree-weight version of Harary index, defined as, $R D D(G)=\frac{1}{2} \sum_{u, v \in V(G)} \frac{d_{G}(u)+d_{G}(v)}{d_{G}(u, v)}$. Alizadeh et al. [1] has shown that the reciprocal degree distance can be used as an efficient measuring tool in the study of complex networks. Hua and Zhang [13] presented some lower and upper bounds of the reciprocal degree distance in terms of graph invariants such as degree distance, Harary index, first Zagreb index, first Zagreb coindex, pendent vertices, independence number, chromatic number, vertex- and edge-connectivity. They also characterized the extremal cactus graphs with the maximum reciprocal degree distance. Alizadeh et al. [1] and Pattabiraman et al. [14, 15] are investigated the behavior of reciprocal degree distance under several standard graph products. It is neccessary and interesting to study this graph invariant.

In these background, Li et al. [18] introduced a vertex-degree-weighted version of $t$ Harary index of a connected graph $G$ called reformulated reciprocal degree distance, which is defined as $\bar{R}_{t}(G)=\frac{1}{2} \sum_{u, v \in V(G)} \frac{d_{G}(u)+d_{G}(v)}{d_{G}(u, v)+t}, t \geq 0$. In view of $\bar{H}_{t}(G), \bar{R}_{t}(G)$ is just the additively weighted $t$-Harary index; while in view of $R D D(G)$ it is also the generalized version of the reciprocal degree distance of a connected graph $G$. It is natural and interesting to study the mathematical properties of this novel graph index.

Li et al. [18] studied the mathematical properties of the reformulated reciprocal degree distance under some edge grafting transformations and extremal properties of the several class of trees. Also they established the sharp upper bound on the maximum reformulated reciprocal degree distance of $n$-vertex trees with $k$ pendents. This motivate for further work by Pattabiraman et al. [16, 17] has obtained the reformulated reciprocal degree distance of some classes of graphs. In this sequence, we have obtained the exact formulae for the reformulated reciprocal degree distance and reciprocal degree distance of disjunction, symmetric difference and Cartesian product of two graphs.

The first Zagreb index is defined as $M_{1}(G)=\sum_{u \in V(G)} d_{G}(u)^{2}=\sum_{u v \in E(G)}\left(d_{G}(u)+d_{G}(v)\right)$. Similarly, the first Zagreb coindex is defined as $\bar{M}_{1}(G)=\sum_{u v \notin E(G)}\left(d_{G}(u)+d_{G}(v)\right)$. The Zagreb indices are found to have applications in QSPR and QSAR studies as well, see [10].

1.1. Disjunction. The disjunction of the graphs $G$ and $H$, denoted by $G \vee H$, has vertex set $V(G) \times V(H)$ and edge set $E(G \vee H)=\{(u, x)(v, y) \mid u v \in E(G)$ (or) $x y \in E(H)\}$. In this section, we obtain the reformulated reciprocal degree distance and reciprocal degree distance of the $G \vee G^{\prime}$. The following lemma follows from the structure of $G \vee H$.

Lemma 1.1. Let $G$ and $H$ be two connected graphs with $n_{1}$ and $n_{2}$ vertices. Then

(i) The distance between two vertices of $G \vee H$ is given by

$d_{G \vee H}((u, x),(v, y))=\left\{\begin{array}{l}1 \text { uv } \in E(G) \text { or } x y \in E(H) \\ 2 \text { otherwise. }\end{array}\right.$

(ii) The degree of $(u, x) \in V(G \vee H)$ is $d_{G \vee H}((u, x))=n_{2} d_{G}(u)+n_{1} d_{H}(x)-d_{G}(u) d_{H}(x)$.

Theorem 1.1. Let $G$ and $H$ be two connected graphs with $n_{1}$ and $n_{2}$ vertices, respectively. Then $\bar{R}_{t}(G \vee H)=\frac{1}{1+t}\left[\left(n_{2}^{3}-4 n_{2} m_{2}\right) M_{1}(G)+\left(n_{1}^{3}-4 n_{1} m_{1}\right) M_{1}(H)+M_{1}(G) M_{1}(H)+\right.$ 
$\left.8 n_{1} n_{2} m_{1} m_{2}\right]+\frac{1}{2+t}\left[\left(n_{2}^{3}-2 n_{2} m_{2}-2 m_{2}\right) \bar{M}_{1}(G)+\left(n_{1}^{3}-2 n_{1} m_{1}-2 m_{1}\right) \bar{M}_{1}(H)-\bar{M}_{1}(G) \bar{M}_{1}(H)+\right.$ $\left.2 n_{1} m_{2}\left(n_{1}^{2}-n_{1}-2 m_{1}\right)+2 n_{2} m_{1}\left(n_{2}^{2}-n_{2}-2 m_{2}\right)\right], t \geq 0$.

Proof. Let $G^{\prime}=G \vee H$. By the definition of reformulated reciprocal degree distance,

$$
\bar{R}_{t}\left(G^{\prime}\right)=\sum_{u, v \in V(G)} \sum_{x, y \in V(H)} \frac{d_{G^{\prime}}((u, x))+d_{G^{\prime}}((v, y)}{d_{G^{\prime}}((u, x),(v, y))+t}
$$

By Lemma 1.1, we have

$$
\begin{aligned}
\bar{R}_{t}\left(G^{\prime}\right)= & \sum_{u, v \in V(G)} \sum_{x, y \in V(H)} \frac{n_{2}\left(d_{G}(u)+d_{G}(v)\right)+n_{1}\left(d_{H}(x)+d_{H}(y)\right)}{d_{G^{\prime}}((u, x),(v, y))+t} \\
& -\sum_{u, v \in V(G)} \sum_{x, y \in V(H)} \frac{\left.\left(d_{G}(u) d_{H}(x)\right)+d_{G}(v) d_{H}(y)\right)}{d_{G^{\prime}}((u, x),(v, y))+t} .
\end{aligned}
$$

We partition the sums into four sums, $S_{1}, S_{2}, S_{3}$ and $S_{4}$ as follows.

$$
\begin{aligned}
S_{1} & =\sum_{u, v \in V(G)} \sum_{x, y \in V(H)}\left\{\frac{d_{G^{\prime}}((u, x))+d_{G^{\prime}}((v, y))}{1+t} \mid x y \in E(H)\right\}, \text { by Lemma } 1.1 \\
& =\frac{1}{1+t}\left(n_{2} m_{2} \sum_{u, v \in V(G)}\left(d_{G}(u)+d_{G}(v)\right)+n_{1} \sum_{u, v \in V(G)} M_{1}(H)-2 n_{1} m_{1} M_{1}(H)\right) \\
& =\frac{1}{1+t}\left(4 n_{1} n_{2} m_{1} m_{2}+n_{1}^{3} M_{1}(H)-2 n_{1} m_{1} M_{1}(H)\right) . \\
S_{2} & =\sum_{u, v \in V(G)} \sum_{x, y \in V(H)}\left\{\frac{d_{G^{\prime}}((u, x))+d_{G^{\prime}}((v, y))}{1+t} \mid u v \in E(G)\right\}, \text { by Lemma } 1.1 \\
& =\frac{1}{1+t}\left(4 n_{1} n_{2} m_{1} m_{2}+n_{2}^{3} M_{1}(G)-2 n_{2} m_{2} M_{1}(G)\right) .
\end{aligned}
$$

By Lemma 1.1, we have

$$
\begin{aligned}
S_{3} & =\sum_{u, v \in V(G)} \sum_{x, y \in V(H)}\left\{\frac{d_{G^{\prime}}((u, x))+d_{G^{\prime}}((v, y))}{1+t} \mid u v \in E(G) \text { and } x y \in E(H)\right\} \\
& =\frac{1}{1+t}\left(2 n_{1} m_{1} M_{1}(H)+2 n_{2} m_{2} M_{1}(G)-M_{1}(G) M_{1}(H)\right) .
\end{aligned}
$$




$$
\begin{aligned}
S_{4}= & \sum_{u, v \in V(G)} \sum_{x, y \in V(H)}\left\{\frac{d_{G^{\prime}}((u, x))+d_{G^{\prime}}((v, y))}{2+t} \mid u v \notin E(G) \text { and } x y \notin E(H)\right\} \\
= & \sum_{u, v \in V(G)} \sum_{x, y \in V(H)}\left\{\frac{d_{G^{\prime}}((u, x))+d_{G^{\prime}}((v, y))}{2+t} \mid u v \notin E(G) \text { and } x y \notin E(H),\right. \\
& u \neq v, x \neq y\} \\
& +\sum_{u v \notin E(G)} \sum_{x \in V(H)} \frac{\left(n_{2}-d_{H}(x)\right)\left(d_{G}(u)+d_{G}(v)\right)+2 n_{1} d_{H}(x)}{2+t} \\
& +\sum_{u \in V(G)} \sum_{x y \notin E(H)} \frac{\left(n_{1}-d_{G}(u)\right)\left(d_{H}(x)+d_{H}(y)\right)+2 n_{2} d_{G}(u)}{2+t} \\
= & \frac{1}{2+t}\left[\left(n_{2}^{3}-2 n_{2} m_{2}-2 m_{2}\right) \bar{M}_{1}(G)+\left(n_{1}^{3}-2 n_{1} m_{1}-2 m_{1}\right) \bar{M}_{1}(H)\right. \\
& \left.-\bar{M}_{1}(G) \bar{M}_{1}(H)+2 n_{1} m_{2}\left(n_{1}^{2}-n_{1}-2 m_{1}\right)+2 n_{2} m_{1}\left(n_{2}^{2}-n_{2}-2 m_{2}\right)\right] .
\end{aligned}
$$

Using $S_{1}$ to $S_{4}$ in (1.1), we have

$$
\begin{aligned}
\bar{R}_{t}\left(G^{\prime}\right)= & S_{1}+S_{2}+S_{4}-S_{3} \\
= & \frac{1}{1+t}\left[\left(n_{2}^{3}-4 n_{2} m_{2}\right) M_{1}(G)+\left(n_{1}^{3}-4 n_{1} m_{1}\right) M_{1}(H)+M_{1}(G) M_{1}(H)\right. \\
& \left.+8 n_{1} n_{2} m_{1} m_{2}\right]+\frac{1}{2+t}\left[\left(n_{2}^{3}-2 n_{2} m_{2}-2 m_{2}\right) \bar{M}_{1}(G)+\left(n_{1}^{3}-2 n_{1} m_{1}-2 m_{1}\right)\right. \\
& \left.\bar{M}_{1}(H)-\bar{M}_{1}(G) \bar{M}_{1}(H)+2 n_{1} m_{2}\left(n_{1}^{2}-n_{1}-2 m_{1}\right)+2 n_{2} m_{1}\left(n_{2}^{2}-n_{2}-2 m_{2}\right)\right] .
\end{aligned}
$$

Using $t=0$, in Theorem 1.1, we obtain the reciprocal degree distance of $G \vee H$.

Corollary 1.1. Let $G$ and $H$ be two connected graphs with $n_{1}, n_{2}$ vertices and $m_{1}, m_{2}$ edges, respectively. Then reciprocal degree distance of $G \vee H$ is $R D D(G \vee H)=\left(n_{2}^{3}-4 n_{2} m_{2}\right) M_{1}(G)+$ $\left(n_{1}^{3}-4 n_{1} m_{1}\right) M_{1}(H)+M_{1}(G) M_{1}(H)+\frac{\left(n_{2}^{3}-2 n_{2} m_{2}-2 m_{2}\right)}{2} \bar{M}_{1}(G)+\frac{\left(n_{1}^{3}-2 n_{1} m_{1}-2 m_{1}\right)}{2} \bar{M}_{1}(H)-$ $\frac{1}{2} \bar{M}_{1}(G) \bar{M}_{1}(H)+n_{1} m_{2}\left(n_{1}^{2}-n_{1}-2 m_{1}\right)+n_{2} m_{1}\left(n_{2}^{2}-n_{2}-2 m_{2}\right)+8 n_{1} n_{2} m_{1} m_{2}$.

One can observe that $M_{1}\left(C_{n}\right)=4 n, n \geq 3, M_{1}\left(P_{1}\right)=0, M_{1}\left(P_{n}\right)=4 n-6, n>1$ and $M_{1}\left(K_{n}\right)=n(n-1)^{2}$. Using Theorem 1.1, we obtain the reformulated reciprocal degree distance of $P_{n} \vee P_{m}, P_{n} \vee C_{m}, C_{n} \vee C_{m}, K_{n} \vee K_{m}, K_{n} \vee P_{m}$ and $K_{n} \vee C_{m}$.

Example 1.1. $(i) \bar{R}_{t}\left(P_{n} \vee P_{m}\right)=\frac{1}{1+t}\left[4 n m\left(n^{2}-6 n+2 n m+m^{2}-6 m+14\right)-6 m\left(m^{2}-\right.\right.$ $\left.4 m+8)-6 n\left(n^{2}-4 n+8\right)+36\right]+\frac{1}{2+t}\left[2 n m\left(n m^{2}-3 m^{2}-6 n m+13 m+n^{2} m-3 n^{2}+13 n-\right.\right.$ $\left.28)+2 m\left(3 m^{2}-11 m+22\right)+2 n\left(3 n^{2}-11 n+22\right)-32\right]$.

(ii) $\bar{R}_{t}\left(K_{n} \vee K_{m}\right)=\frac{1}{1+t}\left[n m(n m-1)^{2}\right]$.

(iii) $\bar{R}_{t}\left(K_{n} \vee P_{m}\right)=\frac{1}{1+t}\left[n m\left(2 n m^{2}-n^{2} m^{2}-m^{2}+6 n^{2} m+2 m-8 n m+4 n^{2}-20 n+20\right)-\right.$ $\left.6 n\left(2 n^{2}-6 n+5\right)\right]+\frac{1}{2+t}\left[n m\left(5 m+n m^{2}-n-m^{2}-10\right)+8 n\right]$. 
Example 1.2. (i) $\bar{R}_{t}\left(P_{n} \vee C_{m}\right)=\frac{1}{1+t}\left[4 n m\left(m^{2}-6 m+n^{2}-4 n+2 n m+8\right)-6 m\left(m^{2}-4 m+\right.\right.$ $4)]+\frac{2}{2+t}\left[n m\left(n m^{2}-3 m^{2}-6 n m+13 m+7 n+n^{2} m-2 n^{2}-14\right)+m\left(3 m^{2}-11 m+10\right)\right]$. (ii) $\bar{R}_{t}\left(C_{n} \vee C_{m}\right)=\frac{1}{1+t}\left[4 n m\left(m^{2}-4 m+n^{2}-4 n+4+2 n m\right)\right]+\frac{1}{2+t}\left[2 n m\left(n m^{2}-2 m^{2}-\right.\right.$ $\left.\left.6 n m+7 m+7 n+n^{2} m-2 n^{2}-6\right)\right]$.

(iii) $\bar{R}_{t}\left(K_{n} \vee C_{m}\right)=\frac{1}{1+t}\left[n m\left(2 n m^{2}-n^{2} m^{2}-m^{2}+6 n^{2} m+2 m-8 n m-4 n^{2}+8 n\right)+4 n^{2}\left(n^{2}-\right.\right.$ $2 n+1)]+\frac{1}{2+t}\left[n m\left(5 m+n m^{2}-3 n m-m^{2}-6\right)\right]$.

Using Corollary 1.1, we obatain the reformulated reciprocal degree distance of $P_{n} \vee$ $P_{m}, P_{n} \vee C_{m}, C_{n} \vee C_{m}, K_{n} \vee K_{m}, K_{n} \vee P_{m}$ and $K_{n} \vee C_{m}$.

Example 1.3. (i) $R D D\left(P_{n} \vee P_{m}\right)=n m\left(m^{2}-11 m+n^{2}-11 n+2 n m+n m^{2}+n^{2} m+28\right)-$ $m\left(3 m^{2}-13 m+26\right)-n\left(3 n^{2}-13 n+26\right)+20$.

(ii) $R D D\left(K_{n} \vee K_{m}\right)=n m(n m-1)^{2}$.

(iii) $R D D\left(K_{n} \vee P_{m}\right)=n m\left(\frac{5 n m^{2}}{2}-n^{2} m^{2}-\frac{3 m^{2}}{2}+6 n^{2} m+\frac{9 m}{2}-8 n m+4 n^{2}-20 n-\frac{n}{2}+\right.$ 15) $-2 n\left(6 n^{2}-18 n+11\right)$.

Example 1.4. (i) $R D D\left(P_{n} \vee C_{m}\right)=n m\left(m^{2}-11 m+2 n^{2}-9 n+2 n m+n m^{2}+n^{2} m+18\right)-$ $m\left(3 m^{2}-13 m+14\right)$.

(ii) $R D D\left(C_{n} \vee C_{m}\right)=n m\left(2 m^{2}-9 m+2 n^{2}-9 n+2 n m+n m^{2}+n^{2} m+10\right)$.

(iii) $R D D\left(K_{n} \vee C_{m}\right)=n m\left(6 n^{2} m-n^{2} m^{2}-\frac{3 m^{2}}{2}+\frac{5 n m^{2}}{2}+\frac{9 m}{2}-\frac{19 n m}{2}-4 n^{2}+8 n-3\right)+$ $4 n^{2}\left(n^{2}-2 n+1\right)$.

1.2. Symmetric difference. For given graphs $G$ and $H$, their symmetric difference $G \oplus H$ is the graph with vertex set $V(G) \times V(H)$ and edge set $E(G \oplus H)=\{(u, x)(v, y) \mid u v \in$ $E(G)$ (or) $x y \in E(H)$ but not both\}. In this section, we obtain the reformulated reciprocal degree distance of the symmetric difference of two connected graphs. Lemma 1.2 follows from the structure of $G \oplus H$.

Lemma 1.2. Let $G$ and $H$ be two connected graphs with $n_{1}$ and $n_{2}$ vertices. Then

(i) The distance between two vertices of $G \oplus H$ is given by $d_{G \oplus H}((u, x),(v, y))=\left\{\begin{array}{l}1 \text { uv } \in E(G) \text { or } x y \in E(H) \text { bot not both } \\ 2 \text { otherwise. }\end{array}\right.$

(ii) The degree of $(u, x)$ in $G \oplus H$ is $d_{G \oplus H}((u, x))=n_{2} d_{G}(u)+n_{1} d_{H}(x)-2 d_{G}(u) d_{H}(x)$.

Using a similar argument as in Theorem 1.1, we have the following theorem.

Theorem 1.2. Let $G$ and $G^{\prime}$ be two connected graphs with $n_{1}$ and $n_{2}$ vertices, respectively. Then $\bar{R}_{t}\left(G \oplus G^{\prime}\right)=\frac{1}{1+t}\left[\left(n_{2}^{3}-7 n_{2} m_{2}\right) M_{1}(G)+\left(n_{1}^{3}-7 n_{1} m_{1}\right) M_{1}(H)+2 M_{1}(G) M_{1}(H)+\right.$ $\left.8 n_{1} n_{2} m_{1} m_{2}\right]+\frac{1}{2+t}\left[\left(n_{2}^{3}-2 n_{2} m_{2}-4 m_{2}\right) \bar{M}_{1}(G)+\left(n_{1}^{3}-2 n_{1} m_{1}-4 m_{1}\right) \bar{M}_{1}(H)-2 \bar{M}_{1}(G) \bar{M}_{1}(H)\right.$ $\left.+2 n_{1} m_{2}\left(n_{1}^{2}-n_{1}-2 m_{1}\right)+2 n_{2} m_{1}\left(n_{2}^{2}-n_{2}-2 m_{2}\right)\right], t \geq 0$.

Using $t=0$, in Theorem 1.2, we obtain the reciprocal degree distance of $G \oplus H$.

Corollary 1.2. Let $G$ and $H$ be two connected graphs with $n_{1}, n_{2}$ vertices and $m_{1}, m_{2}$ edges, respectively. Then reciprocal degree distance of $G \oplus H$ is $R D D(G \oplus H)=\left(n_{2}^{3}-7 n_{2} m_{2}\right) M_{1}(G)+$ $\left(n_{1}^{3}-7 n_{1} m_{1}\right) M_{1}(H)+2 M_{1}(G) M_{1}(H)+\frac{\left(n_{2}^{3}-2 n_{2} m_{2}-4 m_{2}\right)}{2} \bar{M}_{1}(G)+\frac{\left(n_{1}^{3}-2 n_{1} m_{1}-4 m_{1}\right)}{2} \bar{M}_{1}(H)-$ $\bar{M}_{1}(G) \bar{M}_{1}(H)+n_{1} m_{2}\left(n_{1}^{2}-n_{1}-2 m_{1}\right)+n_{2} m_{1}\left(n_{2}^{2}-n_{2}-2 m_{2}\right)+8 n_{1} n_{2} m_{1} m_{2}$. 
Using Theorem 1.2, we obatain the reformulated reciprocal degree distance of $P_{n} \oplus$ $P_{m}, P_{n} \oplus C_{m}, C_{n} \oplus C_{m}, K_{n} \oplus K_{m}, K_{n} \oplus P_{m}$ and $K_{n} \oplus C_{m}$.

Example 1.5. (i) $\bar{R}_{t}\left(P_{n} \oplus P_{m}\right)=\frac{1}{1+t}\left[4 n m\left(n^{2}-9 n+2 n m+m^{2}-9 m+24\right)-6 m\left(m^{2}-\right.\right.$ $\left.7 m+15)-6 n\left(n^{2}-7 n+15\right)+72\right]+\frac{2}{2+t}\left[n m\left(n^{2} m-3 n^{2}-8 n m+19 n+19 m+n m^{2}-\right.\right.$ $\left.\left.3 m^{2}-44\right)+m\left(3 m^{2}-17 m+38\right)+n\left(3 n^{2}-17 n+38\right)-32\right]$.

(ii) $\bar{R}_{t}\left(K_{n} \oplus K_{m}\right)=\frac{1}{1+t}\left[n m\left(-n^{2} m^{2}-\frac{m^{2}}{2}+\frac{5 n m^{2}}{2}+\frac{5 n^{2} m}{2}-\frac{m}{2}-4 n m-\frac{n^{2}}{2}-\frac{n}{2}+2\right)\right]$.

(iii) $\bar{R}_{t}\left(K_{n} \oplus P_{m}\right)=\frac{1}{1+t}\left[n m\left(-\frac{5 n^{2} m^{2}}{2}-\frac{5 m^{2}}{2}+5 n m^{2}+\frac{15 n^{2} m}{2}+\frac{7 m}{2}-11 n m-6 n^{2}+8\right)+\right.$ $\left.3 n\left(n^{2}+n-4\right)\right]+\frac{1}{2+t}\left[n m\left(-2 n m+7 n+7 m+n m^{2}-m^{2}-18\right)-8 n(n-2)\right]$.

Example 1.6. $(i) \bar{R}_{t}\left(P_{n} \oplus C_{m}\right)=\frac{1}{1+t}\left[n m\left(4 m^{2}-36 m+4 n^{2}-28 n+8 n m+60\right)-6 m\left(m^{2}+\right.\right.$ $8)]+\frac{2}{2+t}\left[n m\left(n m^{2}-3 m^{2}-8 n m+19 m+11 n+n^{2} m-2 n^{2}-24\right)+m\left(3 m^{2}-17 m+20\right)\right]$. (ii) $\bar{R}_{t}\left(C_{n} \oplus C_{m}\right)=\frac{1}{1+t}\left[n m\left(4 m^{2}-7 m+4 n^{2}-7 n+8 n m+32\right)\right]+\frac{2}{2+t}\left[n m\left(n m^{2}-2 m^{2}-\right.\right.$ $\left.\left.8 n m+11 m+11 n+n^{2} m-2 n^{2}-12\right)\right]$.

(iii) $\bar{R}_{t}\left(K_{n} \oplus C_{m}\right)=\frac{1}{1+t}\left[n m\left(-\frac{5 n^{2} m^{2}}{2}-\frac{5 m^{2}}{2}+5 n m^{2}+\frac{15 n^{2} m}{2}+\frac{7 m}{2}-11 n m-10 n^{2}+14 n\right)+\right.$ $\left.8 n^{2}\left(n^{2}-2 n+1\right)\right]+\frac{1}{2+t}\left[n m\left(-5 n m+6 n+7 m+n m^{2}-m^{2}-12\right)\right]$.

Using Corollary 1.2, we obtain the reciprocal degree distance of $P_{n} \oplus P_{m}, P_{n} \oplus C_{m}, C_{n} \oplus$ $C_{m}, K_{n} \oplus K_{m}, K_{n} \oplus P_{m}$ and $K_{n} \oplus C_{m}$.

Example 1.7. (i) $R D D\left(P_{n} \oplus P_{m}\right)=n m\left(m^{2}-17 m+n^{2}-17 n+n^{2} m+m^{2} n+52\right)+$ $m\left(-3 m^{2}+25 m-52\right)+n\left(-3 n^{2}+25 n-52\right)+34$.

(ii) $R D D\left(K_{n} \oplus K_{m}\right)=n m\left(-n^{2} m^{2}-\frac{m^{2}}{2}+\frac{5 n m^{2}}{2}+\frac{5 n^{2} m}{2}-\frac{m}{2}-4 n m-\frac{n^{2}}{2}-\frac{n}{2}+2\right)$.

(iii) $R D D\left(K_{n} \oplus P_{m}\right)=n m\left(-\frac{5 n^{2} m^{2}}{2}-3 m^{2}+\frac{11 n m^{2}}{2}+\frac{15 n^{2} m}{2}+7 m-12 n m-6 n^{2}+\frac{7 n}{2}-\right.$ 1) $+n\left(3 n^{2}-5 n+4\right)$.

Example 1.8. (i) $R D D\left(P_{n} \oplus C_{m}\right)=n m\left(m^{2}-17 m+2 n^{2}-17 n+n m^{2}+n^{2} m+36\right)+$ $m\left(-3 m^{2}-17 m-22\right)$.

(ii) $R D D\left(C_{n} \vee C_{m}\right)=n m\left(2 m^{2}+4 m+2 n^{2}+4 n+n m^{2}+n^{2} m+20\right)$.

(iii) $R D D\left(K_{n} \oplus C_{m}\right)=n m\left(-\frac{5 n^{2} m^{2}}{2}-3 m^{2}+\frac{11 n m^{2}}{2}+\frac{15 n^{2} m}{2}+7 m-\frac{27 n m}{2}-10 n^{2}+17 n-\right.$ $6)+8 n^{2}\left(n^{2}-2 n+1\right)$.

1.3. Cartesian product. The Cartesian product, $G \square H$, of graphs $G$ and $H$ has the vertex set $V(G \square H)=V(G) \times V(H)$ and $(u, x)(v, y)$ is an edge of $G \square H$ if $u=v$ and $x y \in E(H)$ or, $u v \in E(G)$ and $x=y$. To each vertex $u \in V(G)$, there is an isomorphic copy of $H$ in $G \square H$ and to each vertex $v \in V(H)$, there is an isomorphic copy of $G$ in $G \square H$. The following lemma follows from the structure of $G \square H$.

Lemma 1.3. Let $G$ and $H$ be two connected graphs with $n_{1}$ and $n_{2}$ vertices, respectively. Then

(i) The distance between two vertices of $G \square H$ is given by $d_{G \square H}\left(\left(u_{i}, v_{j}\right),\left(u_{p}, v_{q}\right)\right)=d_{G}\left(u_{i}, u_{p}\right)+$ $d_{H}\left(v_{j}, v_{q}\right)$.

(ii) The degree of a vertex $\left(u_{i}, v_{j}\right)$ of $G \square H$ is $d_{G}\left(u_{i}\right)+d_{H}\left(v_{j}\right)$.

Now we obtain the lower bound for reformulated reciprocal degree distance of Cartesian product of two connected graphs.

Theorem 1.3. Let $G_{i}$ be the connected graphs with $n_{i}$ vertices and $m_{i}$ edges, $i=1,2$. Then $\bar{R}_{t}\left(G_{1} \square G_{2}\right) \geq n_{2} \bar{R}_{t}\left(G_{1}\right)+n_{1} \bar{R}_{t}\left(G_{2}\right)+4 m_{1} H\left(G_{2}\right)+4 m_{2} H\left(G_{1}\right), t \geq 0$. 
Proof. By the definition of reformulated reciprocal degree distance,

$$
\bar{R}_{t}\left(G_{1} \square G_{2}\right)=\frac{1}{2} \sum_{(u, x),(v, y) \in V\left(G_{1} \square G_{2}\right)} \frac{d_{G_{1} \square G_{2}}((u, x))+d_{G_{1} \square G_{2}}((v, x))}{d_{G_{1} \square G_{2}}((u, x),(v, y))+t} .
$$

By Lemma 1.3, we have

$$
\begin{aligned}
\bar{R}_{t}\left(G_{1} \square G_{2}\right)= & \frac{1}{2} \sum_{(u, x),(v, y) \in V\left(G_{1} \square G_{2}\right)} \frac{d_{G_{1}}(u)+d_{G_{2}}(x)+d_{G_{1}}(v)+d_{G_{2}}(y)}{d_{G_{1}}(u, v)+d_{G_{2}}(x, y)+t} \\
\geq & \frac{1}{2} \sum_{r \in V\left(G_{1}\right)} \sum_{x, y \in V\left(G_{2}\right)}\left(\frac{d_{G_{1}}(u)+d_{G_{1}}(v)}{d_{G_{2}}(x, y)+t}+\frac{d_{G_{2}}(x)+d_{G_{2}}(y)}{d_{G_{2}}(x, y)+t}\right) \\
& +\frac{1}{2} \sum_{u, v \in V\left(G_{1}\right)} \sum_{z \in V\left(G_{2}\right)}\left(\frac{d_{G_{1}}(u)+d_{G_{1}}(v)}{d_{G_{1}}(u, v)+t}+\frac{d_{G_{2}}(x)+d_{G_{2}}(y)}{d_{G_{1}}(u, v)+t}\right) \\
= & n_{2} \bar{R}_{t}\left(G_{1}\right)+n_{1} \bar{R}_{t}\left(G_{2}\right)+4 m_{1} H\left(G_{2}\right)+4 m_{2} H\left(G_{1}\right) .
\end{aligned}
$$

The following corollary gives the reciprocal degree distance of $G_{1} \square G_{2}$.

Corollary 1.3. Let $G_{i}$ be the connected graphs with $n_{i}$ vertices and $m_{i}$ edges, $i=1,2$. Then $R D D\left(G_{1} \square G_{2}\right) \leq n_{2} \bar{R}_{t}\left(G_{1}\right)+n_{1} \bar{R}_{t}\left(G_{2}\right)+4 m_{1} H\left(G_{2}\right)+4 m_{2} H\left(G_{1}\right)$.

1.4. Double graph. Let us denote the double graph of a graph $G$ by $G^{*}$, which is constructed from two copies of $G$ in the following manner. Let the vertex set of $G$ be $V(G)=$ $\left\{v_{1}, v_{2}, \ldots, v_{n}\right\}$, and the vertices of $G^{*}$ are given by the two sets $X=\left\{x_{1}, x_{2}, \ldots, x_{n}\right\}$ and $Y=\left\{y_{1}, y_{2}, \ldots, y_{n}\right\}$. Thus for each vertex $v_{i} \in V(G)$, there are two vertices $x_{i}$ and $y_{i}$ in $V\left(G^{*}\right)$. The double graph $G^{*}$ includes the initial edge set of each copies of $G$, and for any edge $v_{i} v_{j} \in E(G)$, two more edges $x_{i} y_{j}$ and $x_{j} y_{i}$ are added. Now we obtain the $\bar{R}_{t}$ of double graph.

Theorem 1.4. Let $G$ be a connected graph. Then $\bar{R}_{t}\left(G^{*}\right)=8 \bar{R}_{t}(G)+\frac{2 M_{1}(G)}{2+t}$.

Proof. From the structure of the double graph, the distance between two vertices of $G^{*}$ are given as follows.

$d_{G^{*}}\left(x_{i}, x_{j}\right)=d_{G}\left(x_{i}, x_{j}\right), i, j \in\{1,2, \ldots, n\}$.

$d_{G^{*}}\left(x_{i}, y_{j}\right)=d_{G}\left(x_{i}, x_{j}\right), i, j \in\{1,2, \ldots, n\}$.

$d_{G^{*}}\left(x_{i}, y_{i}\right)=2, i \in\{1,2, \ldots, n\}$.

Similarly, the degree of the vertex of $G^{*}$ is $d_{G^{*}}\left(x_{i}\right)=d_{G^{*}}\left(y_{i}\right)=2 d_{G}\left(x_{i}\right), i \in\{1,2, \ldots, n\}$. 


$$
\begin{aligned}
\bar{R}_{t}\left(G^{*}\right)= & \sum_{1 \leq i<j \leq n} \frac{d_{G^{*}}\left(v_{i}\right)+d_{G^{*}}\left(v_{j}\right)}{d_{G^{*}}\left(v_{i}, v_{j}\right)+t} \\
= & \sum_{1 \leq i<j \leq n} \frac{d_{G^{*}}\left(x_{i}\right)+d_{G^{*}}\left(x_{j}\right)}{d_{G^{*}}\left(x_{i}, x_{j}\right)+t}+\sum_{1 \leq i<j \leq n} \frac{d_{G^{*}}\left(y_{i}\right)+d_{G^{*}}\left(y_{j}\right)}{d_{G^{*}}\left(y_{i}, y_{j}\right)+t} \\
& +\sum_{i, j=1, i \neq j}^{n} \frac{d_{G^{*}}\left(x_{i}\right)+d_{G^{*}}\left(y_{j}\right)}{d_{G^{*}}\left(x_{i}, y_{j}\right)+t}+\sum_{i=1}^{n} \frac{d_{G^{*}}\left(x_{i}\right)+d_{G^{*}}\left(y_{i}\right)}{d_{G^{*}}\left(x_{i}, y_{i}\right)+t} \\
= & \sum_{1 \leq i<j \leq n} \frac{2 d_{G}\left(x_{i}\right)+2 d_{G}\left(x_{j}\right)}{d_{G}\left(x_{i}, x_{j}\right)+t}+\sum_{1 \leq i<j \leq n} \frac{2 d_{G}\left(x_{i}\right)+2 d_{G}\left(x_{j}\right)}{d_{G}\left(x_{i}, x_{j}\right)+t} \\
& +\sum_{i, j=1, i \neq j}^{n} \frac{2 d_{G}\left(x_{i}\right)+2 d_{G}\left(x_{j}\right)}{d_{G}\left(x_{i}, x_{j}\right)+t}+\sum_{x_{i} \in V(G)} \frac{2 d_{G}\left(x_{i}\right)+2 d_{G}\left(x_{i}\right)}{2+t} \\
= & 2 \bar{R}_{t}(G)+2 \bar{R}_{t}(G)+4 \bar{R}_{t}(G)+\frac{2}{2+t} \sum_{x_{i} \in V(G)} d_{G}^{2}\left(x_{i}\right) \\
= & 8 \bar{R}_{t}(G)+\frac{2 M_{1}(G)}{2+t} .
\end{aligned}
$$

If we consider $t=0$, in the above theorem, we have the following corollary.

Corollary 1.4. Let $G$ be a connected graph. Then $R D D\left(G^{*}\right)=8 R D D(G)+M_{1}(G)$.

By direct calculations we obtain expressions for the values of the Harary indices of $P_{n}$ and $C_{n} . H\left(P_{n}\right)=n\left(\sum_{i=1}^{n} \frac{1}{i}\right)-n$ and $H\left(C_{n}\right)=\left\{\begin{array}{l}n\left(\sum_{i=1}^{\frac{n}{2}} \frac{1}{i}\right)-1, \text { if } \mathrm{n} \text { is even } \\ n\left(\sum_{i=1}^{\frac{n-1}{2}} \frac{1}{i}\right), \text { if } \mathrm{n} \text { is odd. }\end{array}\right.$

The following are the reciprocal degree distance for complete graph, path and cycle on $n$ vertices by direct calculations: $R D D\left(K_{n}\right)=n(n-1)^{2}, R D D\left(P_{n}\right)=H\left(P_{n}\right)+4\left(\sum_{i=1}^{n-1} \frac{1}{i}\right)-$ $\frac{3}{n-1}$ and $R D D\left(C_{n}\right)=4 H\left(C_{n}\right)$.

Using Corollary 1.4, we obtain the reciprocal degree distance of double graph of $K_{n}, P_{n}$ and $C_{n}$.

Example 1.9. (i) $R D D\left(K_{n}^{*}\right)=9 n(n-1)$.

(ii) $R D D\left(P_{n}^{*}\right)=4(2 n+1)\left(\sum_{i=1}^{n-1} \frac{1}{i}\right)-4 n-\frac{3}{n-1}+2$.

(iii) $R D D\left(C_{n}^{*}\right)=\left\{\begin{array}{l}32 n\left(\sum_{i=1}^{n} \frac{1}{i}-1\right)+4 n \text { if } n \text { is even } \\ 32 n\left(\sum_{i=1}^{\frac{n-1}{2}} \frac{1}{i}\right)+4 n \text { if } n \text { is odd. }\end{array}\right.$

\section{REFERENCES}

[1] Alizadeh, Y., Iranmanesh, A. and Doslic, T., Additively weigthed Harary index of some composite graphs, Discrete Math., 313 (2013), 26-34

[2] Berinde, Z. M., Comparing the molecular graph degeneracy of Wiener, Harary, Balaban, Randic and ZEP topological indices, Creat. Math. Inform., 23 (2014), 165-174 
[3] Berinde, Z. M., QSTR mathematical models for the toxicity of aliphatic carboxylic acids on tetrahymena pyriformis, Creat. Math. Inform., 22 (2013), 151-160

[4] Berinde, Z. M., A QSPR study of hydrophobicity of phenols and 2-(aryloxy- $\alpha$-acetyl)-phenoxathiin derivatives using the topological index ZEP, Creat. Math. Inform., 22 (2013), 33-40

[5] Berinde, Z. M., Modelling normal boiling points of alkanes by linear regression using the SD index, Creat. Math. Inform., 19 (2010), 135-139

[6] Crucitti, P., Latora, V. and Porta, S., Centrality measures in spatial network of Urban streets, Phys. rev. E, 73 (2006), 036125

[7] Das, K. C., Zhou, B. and Trinajstić, N., Bounds on Harary index, J. Math. Chem., 46 (2009), 1369-1376

[8] Das, K. C., Xu, K. X., Cangul, I. N., Cevik, A. S. and Graovac, A., On the Harary index of graph operations, J. Inequal. Appl., 16 (2013), 2013-339

[9] Dobrynin, A. A. and Kochetova, A. A., Degree distance of a graph: a degree analogue of the Wiener index, J. Chem. Inf. Comput. Sci., 34 (1994), 1082-1086

[10] Devillers, J. and Balaban, Eds. A. T., Topological indices and related descriptors in QSAR and QSPR, Gordon and Breach, Amsterdam, The Netherlands, 1999

[11] Gutman, I., Selected properties of the Schultz molecular topological index, J. Chem. Inf. Comput. Sci., 34 (1994), 1087-1089

[12] Gutman, I. and Polansky, O. E., Mathematical Concepts in Organic Chemistry, Springer-Verlag, Berlin, 1986

[13] Hua, H. and Zhang, S., On the reciprocal degree distance of graphs, Discrete Appl. Math., 160 (2012) 1152-1163

[14] Pattabiraman, K. and Vijayaragavan, M., Reciprocal degree distance of product graphs, Discrete Appl. Math., 179 (2014), 201-213

[15] Pattabiraman, K. and Vijayaragavan, M., Reciprocal degree distance of some graph operations, Trans. Comb., 2 (2013), 13-24

[16] Pattabiraman, K. and Vijayaragavan, M., Reformulated reciprocal degree distance of transformation graph, Electronic Notes in Discrete Math, (in press)

[17] Pattabiraman, K. and Vijayaragavan, M., Reformulated reciprocal degree distance of graph operations, Int. J. Appl. Math. Comput., DOI: 10.1007/s40819-016-0159-6

[18] Shuchao, Li., Yueyu, Wu. and Zhang, H., On the reformulated reciprocal sum-degree distance of graph transformations, Discrete Appl. Math., 193 (2015), 162-173

[19] Yousefi-Azari, H., Khalifeh, M. H. and Ashrafi, A. R., Calculating the edge Wiener and edge Szeged indices of graphs, J. Comput. Appl. Math., 235 (2011), 4866-4870

\author{
DEPARTMENT OF MATHEMATICS \\ ANNAMALAI UNIVERSITY \\ ANNAMALAINAGAR 608 002, INDIA \\ Email address: pramank@gmail. com \\ DEPARTMENT OF MATHEMATICS \\ Thiruvalluvar College OF ENGINEERING AND TECHNOlOGY \\ VANDAVASI 604 505, INDIA \\ Email address: mvragavano9@gmail.com
}

